\title{
High Resolution X-ray Spectroscopy of
}

Cosmic Plasmas

\section{.}

\section{IAU COLLOQUIUM 115}

\section{EDITED BY PAUL GORENSTEIN IND MARTIN ZOMBECK}


High Resolution X-ray Spectroscopy of Cosmic Plasmas 
International Astronomical Union

Union Astronomique Internationale

The following Colloquia of the International Astronomical Union are published for the Union by Cambridge University Press.

82. Cepheids. Edited by Barry F. Madore. 0521300916.1985

91. History of Oriental Astronomy. Edited by G. Swarup, A.K. Bag and K.S.Shukla. 052134659 2. 1987

92. Physics of Be Stars. Edited by A. Slettebak and T.P. Snow. 0521330785.1987

101. Supernova Remnants and the Interstellar Medium. Edited by R.S. Roger and T.L. Landecker. 052135062 X. 1988

105. The Teaching of Astronomy. Edited by Jay M. Pasachoff and John R. Percy. 0521353319.1990

106. Evolution of Peculiar Red Giant Stars. Edited by Hollis Johnson and Ben Zuckerman 052136617 8. 1989

111. The Use of Pulsating Stars in Fundamental Problems of Astronomy. Edited by Edward G. Schmidt 052137023 X. 1989

115. High Resolution X-ray Spectroscopy of Cosmic Plasmas. Edited by Paul Gorenstein and Martin Zombeck 0521370183.1990 
International Astronomical Union

Union Astronomique Internationale

\section{High Resolution X-ray Spectroscopy of Cosmic Plasmas}

The proceedings of International Astronomical Union 115th colloquium

Edited by

PAUL GORENSTEIN AND MARTIN ZOMBECK

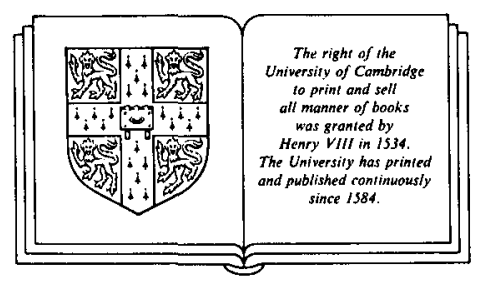

CAMBRIDGE UNIVERSITY PRESS

Cambridge

New York Port Chester

Melbourne Sydney 
Published by the Press Syndicate of the University of Cambridge The Pitt Building, Trumpington Street, Cambridge CB2 1RP

40 West 20th Street, New York, NY 10011, USA

10 Stamford Road, Oakleigh, Melbourne 3166, Australia

(C) Cambridge University Press 1990

First published 1990

Printed in Great Britain at the University Press, Cambridge

British Library cataloguing in publication data

Library of Congress cataloguing in publication data

ISBN 0521370183 


\section{CONTENTS}

Organizing Committees viii

Acknowledgements viii

Preface $\quad$ ix

Colloquium Photograph $\quad$ x

List of Participants $\quad x i$

1. X-rays from a Hot Plasma

Emission Lines From Hot Astrophysical Plasmas

J.C. Raymond

Spectroscopic Diagnostics for Ions Observed in Solar and Cosmic Plasmas

H. Mason

Recent Advances in Atomic Modelling W. Goldstein

Relativistic Free-free Gaunt Factor of the Dense High Temperature Stellar Plasma

N. Itoh, M. Nakagawa and Y. Kohyama

The Differential Emission Measure of $\lambda$ And

M. Landini, B.C. Monsignori Fossi and R. Pallavicini

$\mathrm{X}$-ray Lines From Mg VIII and Si X Ions and Their Diagnostic Use

B.N. Dwivedi

Thermal Instability in a Hot Plasma

S.A. Balbus and N. Soker

$\mathrm{X}$-ray Line Formation in Astrophysical Environments: The L-shell, Spectra of Ionized Iron

D.A. Liedahl, S.M. Kahn, A.L. Osterheld and W.H. Goldstein

Measurement of Carbon Ion Photoabsorption Cross Sections Using Laser Plasmas

B.J. Wargelin, S.M. Kahn, W.W. Craig and R. London

Tokomak Plasmas: A Paradigm for Coronal Equilibrium and Disequilibrium

R. Petrasso

2. Magnetic Effects

Low Energy Lines in Spectra of Gamma Ray Bursts

G. Bisnovatyi-Kogan and A.F. Illarionov

The Effects of Magnetic Fields Upon Cosmic X-ray Spectra

P. Mészáros

Effect of Vacuum Polarization in a Strong Magnetic Field and Spectral Features of X-Ray Source

Emission

Y. Gnedin

Merging of Iron Lines in Spectra of X-ray Burst Sources J. Madej

Role of Magnetospheric Plasma Physics for Understanding Cosmic Phenomena

I.M.L. Das

\section{Stellar Coronae}

Goals for the Application of High Resolution X-ray Spectroscopy to the Diagnosis of Stellar Corona Plasmas

J.L. Linsky

X-ray Spectroscopy across the HR Diagram

J.H.M.M. Schmitt

Spectroscopy of Stellar Coronal Sources with the Medium Energy Experiment on EXOSAT

R. Pallavicini, L. Pasquini, J.H.M.M. Schmitt and G. Tagliaferri

$\mathrm{X}$-ray Spectral Synthesis in Hydrodynamic Flare Models

S. Serio, E. Antonucci, M.A. Dodero, G. Peres and F. Reale

$X$-ray Spectral Diagnostics for Coronal Loops in the Active K Dwarf AB Doradus

O. Vilhu and A.C. Cameron 
4. Supernova Remnants, Soft X-ray Background

High Resolution Spectroscopy and Plasma Diagnostics of Supernova Remnants

C. Canizares

Features in the Soft X-ray Background

R. Rothenflug

Non-equilibrium Ionisation in Tycho's Supernova Remnant

W. Brinkmann

Fe VIII, IX, and X Line Emission From the Soft X-ray Background: Previous Limits and a Future Measurement

J.J. Bloch, W.C. Priedhorsky and B.W. Smith

$\mathrm{X}$-ray emission from Reverse-Shocked Ejecta in Supernova Remnants

D.F. Cioffi and C.F. McKee

Integrated X-ray Surface Brightnesses of Supernova Remnants and Comparison with Radio and Infrared Values

J.R. Dickel, L.K. Norton and P.J. Gensheimer

A Comparison of Three Regions of Puppis A

M.F. Fischbach, L.M. Bateman, C.R. Canizares, T.H. Markert and P.J. Saez

\section{Compact Binaries}

An EXOSAT Observation of Spectral Variability From RS CVn Binary AR LAC

N.E. White, R.A. Schafer, A.N. Parmer, K. Horne and J.L. Culhane

Theory of Accretion Disk Coronae

T. Kallman

Lin Structures in the X-ray Spectra of Cygnus X-2 Observed With EXOSAT

P.E. Freeman, S.M. Kahn, L. Chiapetti, A. Ciapi, L. Maraschi, E.G. Tanzi, A. Treves,

E.G. Branduardi-Raymont and E.N. Ercan

X-ray Absorption by Ionized Gas in EXOSAT Spectra from the Binary System 4U1700-37/HD153919

F. Haberl, T.R. Kallman and N.E. White

X-ray Spectroscopy of the Ultra-soft Transient 4U1543-47

H. van der Woerd, N.E. White and S.M. Kahn

6. Clusters of Galaxies, Cooling Flows

$\mathrm{X}$-ray Spectra of Clusters of Galaxies

C. Sarazin

Signatures of Cooling Flows

A. Fabian

Alternatives to the Existence of Large Cooling Flows

W. Tucker

Evolution of the Coronae in Early Type Galaxies

L. David, W. Forman and C. Jones

Implications of Abundance Measurements of the Intracluster Medium

W. Forman, C. Jones and L. David

Clusters of Galaxies and the Hot Intracluster Medium

C. Jones, W. Forman and L. David

The Evolution of Cooling Flow and the Mass Deposition Process

M. Hattori and A. Habe

A Cooling Flow Cluster at Redshift, $\mathrm{Z}=0.2$

A. Wolter, I.M. Gioia, T. Maccacaro, S.L. Morris, R. Nesci, G.C. Perola and R.E. Schild

\section{Active Galactic Nuclei, Cosmic X-ray Background}

High Resolution X-ray Spectroscopy of Active Galactic Nuclei J. Krolik

Comsic X-ray Background from Early Active Galactic Nuclei

A. Zdziarski

Cosmic X-ray Background from Self-absorbed Low Luminosity AGNs

J.E. Grindlay and M. Luke 


\section{Future X-ray Observatories}

Comments on the Future Observatories and Their X-ray Spectroscopy Capability

J.L. Culhane

The ROSAT Mission

J. Trümper

The SPEKTROSAT Mission

P. Predehl

The X-ray Astronomy Satellite SAX

R.C. Butler

XSPECT: A Telescope/Spectrometer System on SPECTRUM-RÖNTGEN-GAMMA

H. Schnopper

JET-X: A Joint European X-ray Telescope for SPECTRUM-X

A. Wells, D.H. Lumb, K..A. Pounds, G.C. Stewart, B. Aschenbach, H. Brauninger, G. Hasinger,

J. Trümper, O. Citterio, L. Scarsi, A. Peacock and B. Taylor

9. Future X-ray Observatories, Detectors and Instrumentation

A High Resolution Echelle Spectrometer for Soft X-ray and EUV Astronomy

$\mathrm{J}$. Green and S. Bowyer

Expected Scientific Performance of the Three Spectrometers on the Extreme Ultraviolet Explorer

J. Vallerga, P. Jelinsky, P.W. Vedder and R. Malina

Dispersive Spectroscopy on AXAF

T. Markert

Thermal Detectors for X-ray Astronomy

S. Holt

Innovative Techniques for X-ray Calorimetry

S. Labov, E. Silver, D. Landis, N. Madden, F. Goulding, J. Beeman, E. Haller, J. Rutledge,

G. Bernstein and P. Timbie

Observing Soft X-ray Line Emission from the Interstellar Medium with X-ray Calorimeter on a

Sounding Rocket

J. Zhang, B. Edwards, M. Juda, R. Kelley, G. Madejski, D. McCammon, H. Moseley, M. Skinner,

R. Schoelkopf and A. Szymkowiak

High Throughput Soft X-ray Spectroscopy With Reflection Gratings

S. Khan

The SHEAL Diffuse X-ray Spectrometer Experiment

W.T. Sanders, S.L. Snowden and R.J. Edgar

Stigmatic Spectroscopic Instruments for the Wavelength Range 30-300 § With High Angular and Spectral Resolution Using Multi-layer Mirrors

E.N. Ragozin

Object Index 